\section{Postwachstumsökonomie und nachhaltige Entwicklung - Zwei (un)vereinbare Ideen?}

\author{
von Jürgen Kopfmüller und Linda Nierling, \\ Institut für Technikfolgenabschätzung und \\ Systemanalyse (ITAS), Karlsruhe, André \\ Reichel, Karlshochschule International \\ University, Karlsruhe, und Marius Albiez, \\ Eberhard Karls Universität Tübingen
}

Insbesondere seit der Wirtschafts- und Finanzkrise von 2008 haben wachstumskritische Initiativen und Publikationen erheblich an Zahl und öffentlicher Sichtbarkeit zugenommen. Sie positionieren sich dabei vielfach kritisch zu den seit rund $\mathbf{3 0}$ Jahren laufenden und in verschiedener Hinsicht etablierten Debatten und Politiken einer nachhaltigen Entwicklung. In diesem Beitrag werden die verschiedenen Facetten des Postwachstumsbegriffs skizziert, und es wird anhand der aktuellen Auseinandersetzungen sowie zweier empirischer Beispiele der Frage nachgegangen, wie sich das Postwachstums- und das Nachhaltigkeitsleitbild zueinander verhalten, und wo gemeinsame Potenziale eines Beitrags zur Realisierung der erforderlichen gesellschaftlichen Transformationen liegen können.

In recent years, particularly since the economic and financial crisis in 2008, critical initiatives and publications towards growth have increased in numbers but also in their public visibility. These initiatives hereby very often took a critical position towards the debates and politics of sustainable development which have been going on for 30 years. In this contribution, different facets of the understanding of "postgrowth" are analysed. On the basis of the current state of debate, as well as two empirical examples, the question will be examined, how growth-critical ideas relate to sustainable development and where potential similarities might lead to a join contribution to envisioned societal transformation processes.

\section{Einleitung}

Spätestens seit der 4. Internationalen DegrowthKonferenz 2014 in Leipzig mit mehr als 3.000 TeilnehmerInnen ist der Begriff „Degrowth“ in der deutschen Community fest verankert. Dabei ist die hohe öffentliche Aufmerksamkeit für das Thema ebenso faszinierend wie die frischen Ideen, die von unten Wege in eine ökonomische, ökologische und soziale Transformation intendieren. Allerdings hat sich der Postwachstumsdiskurs von Anfang an als ein Gegenentwurf zu dem inzwischen etablierten Konzept einer nachhaltigen Entwicklung verstanden. Diese Distanz beider Ansätze ist auf den ersten Blick nachzuvollziehen, da sonst eine eigenständige Positionierung von „Postwachstum“ kaum möglich gewesen wäre. Auf den zweiten Blick überrascht allerdings die starke Trennung der beiden Diskussionsstränge, da es auf inhaltlicher Ebene eine geteilte Zielorientierung hin zu der Gestaltung einer ökologisch nachhaltigen und sozial gerechten Zukunft gibt.

In diesem Beitrag wird die derzeitig von einigen konstatierte Unvereinbarkeit beider Debatten nachvollzogen. Hierfür wird zunächst der Postwachstumsbegriff selbst einer Positionsbestimmung unterzogen, um die Vielschichtigkeit der Verwendung unterschiedlicher Verständnisse von Postwachstum darzustellen (Kap. 2). Im Anschluss wird dargelegt, dass es zwar eine Reihe von Argumenten gibt, die für die Unvereinbarkeit beider Leitbilder sprechen. Allerdings zeigt sich auf konzeptioneller Ebene auch eine Reihe von Gemeinsamkeiten beider Diskurse, die auf ihre starke innere Verzahnung hinweist (Kap. 3). Auf Basis dieser konzeptionellen Überlegungen werden im Anschluss anhand einer öffentlichen Auseinandersetzung von Andrew Stirling (Vertreter der Nachhaltigkeitsposition) und Giorgos Kallis (Vertreter der Degrowth-Position) mögliche Konfliktlinien nachvollzogen, aus denen einige die These der Unvereinbarkeit ableiten. Im Anschluss illustriert ein Beispiel aus der ReallaborForschungspraxis der AutorInnen, dass auf einer (forschungs-)praktischen Ebene Handlungsfelder aus den Bereichen „Nachhaltigkeit" und „Postwachstum" oft nicht zu trennen sind. Der Artikel schließt mit einer Reflexion über mögliche Funktionen und negative Implikationen der Trennung der beiden Diskurse, plädiert für eine sorgfältige Analyse der Beweggründe und Ursprünge, die zu den derzeitigen noch immer stark postulierten Unvereinbarkeiten geführt haben und zeigt Möglichkeiten auf, sie zu überwinden (Kap. 4). 


\section{Facetten des Postwachstumsbegriffs}

Seit der französische Philosoph Serge Latouche im Jahr 2004 den Begriff der „Décroissance“ als „Wortgranate“ - er spricht von „mot-obus“ - in den Nachhaltigkeitsdiskurs geworfen hat (Latouche 2004), kann von einer Renaissance wachstumskritischer Betrachtungen und Positionen gesprochen werden, die es in dieser Intensität und Form seit den 1970ern nicht mehr gegeben hat. Auf Englisch als „Degrowth“ bezeichnet, hat sich im deutschsprachigen Raum die Bezeichnung „Postwachstumsökonomie“ durchgesetzt und lässt sich auf den Oldenburger Wirtschaftswissenschaftler Niko Paech zurückführen (Paech 2005; Paech 2009). Seit 2009 wird aber auch im Englischen von ,postgrowth“ oder ,post growth“ gesprochen. In einem Artikel in der Harvard Business Review spricht James Spaeth von einer

,postgrowth world where working life, the natural environment, communities, and the public sector will no longer be sacrificed for the sake of mere GDP growth." (Spaeth 2009)

Im selben Jahr wurde auch das Post Growth Institute als offenes Netzwerk von Forschenden und Bewegungsaktiven gegründet. Es dient als Zentrum und Vernetzer von Postwachstumsinstitutionen im anglo-amerikanischen Raum mit seiner „Post Growth Alliance“. Ein Schwerpunkt der Forschungsarbeit in den letzten Jahren waren Untersuchungen im Not-for-profit-Sektor, insbesondere zu dessen Organisationsformen, Geschäftsmodellen und Managementansätzen (Maclurcan/Hinton 2014). Auf dessen Webseite wird „Postgrowth“ als Oberbegriff bezeichnet, der für eine Vielfalt an Konzepten steht, die sich mit Gesellschaften ,nach der Wachstumsstory“ befasst (Post Growth Institute 2016). Wenn die Definition von Décroissance und Degrowth im Latoucheschen Sinne dagegen gestellt wird, kommen die Unterschiede in den Blick. Auf der Internetpräsenz des Forschungsnetzwerks zu Degrowth, dem zentralen europäischen Netzwerk der DegrowthBewegung, erscheint der Begriff als „downscaling of production and consumption that increases human well-being and enhances ecological conditions and equity on the planet" (Research \& Degrowth 2016). Die Postwachstumsökonomie in dieser Lesart fokussiert auf ökologische Beschei- denheit, offene und lokale Wirtschaftskreisläufe und eine gerechtere Verteilung von Ressourcen durch neue Formen demokratischer Institutionen. Dies ist eine engere und detaillierte Formulierung einer Postwachstumsökonomie als es der weitere Postgrowth-Begriff nahe legt. Die Vielfalt an Postwachstumszukünften scheint dabei weiter definiert zu sein als das Verständnis von Degrowth und lässt letzteres Konzept als einen konkreten Ansatz unter dem Dach von Postgrowth erscheinen. Es lassen sich wohl einige grundlegende Gemeinsamkeiten aller Ansätze unter dem Postgrowth-Dach festhalten, unter anderem der Abschied von einer BIP-orientierten Definition wirtschaftlichen und gesellschaftlichen Wohlstands inklusive einer Neuorientierung der Wirtschaftspolitik auf geeignetere Wohlfahrtsmaße. Ebenso wird die Auffassung geteilt, dass die natürlichen Ökosysteme eine harte Begrenzung und Rahmung menschlicher Aktivitäten darstellen. Bei der Frage des Wachstums ist zumindest beim Postgrowth-Begriff die Unterscheidung zwischen gutem und schlechtem Wachstum vorhanden, die an Herman E. Daly erinnert und seine Unterscheidung zwischen ,„ökonomischem Wachstum“ und „unökonomischem Wachstum“ (Daly/Farley 2003). Zentral bei Postgrowth ist der Fokus auf die menschliche Dimension, den Vorrang des Menschen vor der Wirtschaft, ein beinahe aristotelisches Verständnis von „Oikonomia“.

Der Hinweis auf die sprachlichen Unterscheidungen ist dabei nicht sophistisch motiviert, sondern ist aus Sicht der AutorInnen wichtig für die Einschätzung der Debatte um Wachstum oder Postwachstum. Die wachstumskritische Debatte hat inzwischen eine große Ausdifferenzierung erfahren, sowohl hinsichtlich der Motive der Beteiligten als auch der Identifikation der konzeptionellen Grundlagen und praktischen Handlungsfelder (D'Alisa et al. 2015; Eversberg/Schmelzer 2016). In Deutschland wird dabei seit einiger Zeit über Unternehmen als Postwachstumspioniere geforscht, was in der Degrowth-Bewegung bisher eher als randständiges Thema betrachtet wurde (Liesen et al. 2013; Reichel 2013). Gleichzeitig kann eine zu enge Definition von „Postgrowth“ letztlich die Gefahr einer zu starken Schließung und Verengung der Debatte und eines Verlusts an diskursiver Flexibilität bergen. Es wäre da- 
her die im deutschen Sprachraum unscharfe Vermischung von Postgrowth und Degrowth zu reflektieren und zwischen Postwachstum im weiteren Sinne, verstanden als Dachbegriff, und Postwachstum im engeren Sinne, verstanden als Degrowth und spezielles Postwachstumskonzept, zu unterscheiden. Eine solche Unterscheidung erscheint umso wichtiger, als auch eine Positionsbestimmung zwischen Nachhaltiger Entwicklung und Postwachstum noch nicht abschließend vorgenommen wurde. In der Degrowth-Perspektive erscheint nämlich Nachhaltige Entwicklung als hegemoniales Konzept der Strukturbewahrung, das sein Ziel - eine dauerhaft ökologisch tragfähige, sozial gerechte und inklusive wirtschaftliche Entwicklung für die gesamte Menschheit nicht erreichen kann (Martínez-Alier et al. 2010).

\section{Das Verhältnis zwischen Degrowth und nachhaltiger Entwicklung}

Die Kritik aus der Degrowth-Community, hier im engeren Verständnis von Degrowth, am Leitbild der Nachhaltigen Entwicklung speist sich aus wenigstens drei Quellen. Zum einen wird das faktische Versagen der Umsetzung von Nachhaltiger Entwicklung angeklagt. Die ökologische Lage des Planeten ist in manchen Bereichen schlechter als vor 30 Jahren, als der Brundtland-Bericht seine berühmte Nachhaltigkeitsdefinition formulierte. Dies gilt bezogen auf den ökologischen Fußabdruck (WWF 2014), die planetaren Grenzen (Rockström et al. 2009) oder den Treibhausgasausstoß. Was dabei aber nicht ausreichend Beachtung findet, sind die Fortschritte auf anderen Feldern, wie die fast komplette Eliminierung der Emission ozonabbauender Stoffe oder erhebliche Verbesserungen bei der Trinkwasser- und Sanitärversorgung für Millionen Menschen in Entwicklungsländern (UN 2015a). In der ökonomischen Sphäre hat die Finanzkrise von 2008/2009 und ihre Tiefen- und Langzeitwirkungen erneut deutlich gemacht, dass auch das ökonomische System äußerst fragil und vulnerabel ist. Auch hier sollte jedoch nicht übersehen werden, dass in den vergangenen Jahren für Millionen Menschen Erfolge bei der Bekämpfung von Armut und Hunger, bei der Verbesserung der Grundbildung oder bei der
Bekämpfung von Krankheiten wie Malaria oder Tuberkulose erreicht wurden. (UN 2015a).

Eine zweite Quelle der Kritik bezieht sich auf das Entwicklungsparadigma, welches seit Harry S. Trumans berühmter Inaugurationsrede 1949 mit Auslandsinvestitionen, Technologietransfer und wachsenden Einkommen gleichgesetzt wird (Truman 1949)

Entwicklung wird aus Degrowth-Perspektive als eine neue Form des Kolonialismus, als Ausdehnung westlicher Hegemonie über den globalen Süden gebrandmarkt. Hier muss allerdings eingewendet werden, dass sich kaum politische Stimmen aus dem Süden finden, die von einer notwendigen Abkehr vom Entwicklungsgedanken oder einer „Ent-Entwicklung“ sprechen. Gerade die G77-Staaten fordern seit Jahren eine globale Entwicklungspolitik, die stärker durch das Prinzip der Gleichberechtigung gekennzeichnet ist. Eine Reform der weltwirtschaftlichen Rahmenbedingungen, etwa im Bereich der Handelspolitik durch die stärkere Öffnung der Märkte Europas und Nordamerikas für Entwicklungsländer, ist Teil dieser Forderung (Group of 77 2015). In der entwicklungspolitischen Debatte und Praxis begann zudem seit den 1990er Jahren jenseits der lange Zeit dominierenden Kontroverse zwischen Dependenz- und Modernisierungstheorie (Stockmann et al. 2015) eine Transformation des Entwicklungsdiskurses, die mit den Begriffen Good Governance (UN-ESCAP 2009), Global Governance (Zürn 1998, Willke 2006) oder zivilgesellschaftliche Partizipation und mit dem Versuch verbunden ist, eine gerechtere und den spezifischen Problemlagen angemessenere globale Ordnung zu entwickeln. Dieser Prozess kann auch als eine Folge der Debatten um nachhaltige Entwicklung gesehen werden.

Schließlich wird dem Konzept Nachhaltiger Entwicklung vorgeworfen, dass es letztlich nur eine Verschleierung des Wachstumsparadigmas mit anderen Mitteln sei, da Entwicklung immer Einkommensentwicklung im Sinne von Steigerung meint. Gleichzeitig würde mit Nachhaltiger Entwicklung einer absoluten Entkopplung von Umweltverbrauch und Wirtschaftswachstum das Wort geredet, die aus Degrowth-Sicht ein reiner Mythos ist. In der Tat ist gegen mehr Vorsicht bei der Hoffnung auf die Entkopplungswet- 
te der Proponenten des „Grünen Wachstums“ oder einer "Green Economy“, zu denen insbesondere das Umweltprogramm der Vereinten Nationen UNEP zählt, nichts einzuwenden. Die Empirie ist unklar (Binswanger et al. 2005), die Entkopplungsmodelle vage (Bleischwitz et al. 2012) und aus verantwortungsethischer Sicht ist eher eine vorsichtige Haltung gegenüber der Entkopplungsvorstellung angebracht (Grunwald 2015). Die Nachhaltigkeitsidee als verschleiertes Wachstumsparadigma zu bezeichnen, ist aber zumindest aus zwei Gründen unangemessen. Zum einen wurde bereits im Brundtland-Bericht eine durchaus differenzierte Haltung zum Wachstum eingenommen. Wachstum galt dort als eingebettet in die Prinzipien der Nachhaltigkeit - genauer: der Einhaltung ökologischer Belastungsgrenzen und der langfristigen Ressourcenzugangsgerechtigkeit sowie der Sicherung der Bedürfnisbefriedigung der Ärmsten der Welt - und der Nichtausbeutung Dritter (WCED 1987, Kap. 2.I.6). Apodiktisch wird gleich im Anschluss an diese Aussage formuliert: „But growth is not enough." Zum anderen zeigt die Debatte um die Effizienz-Konsistenz-Suffizienz-Trias in der Frage nach geeigneten Strategien zur Erreichung nachhaltiger Entwicklung, dass - wenngleich de facto verkürzt auf die ökologische Dimension wachstumskritische Ansätze hier von Beginn an mitgedacht wurden (etwa Huber 1995).

Gleichzeitig hat das Konzept Nachhaltige Entwicklung, in einem größeren Zusammenhang gedacht, herausragende Erfolge zumindest auf drei Ebenen vorzuweisen, an die sich eine breitere Postwachstumsdiskussion anknüpfen lässt.

Erstens ist Nachhaltige Entwicklung ein globaler Referenzrahmen für politisches Handeln geworden. Gerade durch die Formulierung und Verabschiedung der Sustainable Development Goals (SDG) durch die Vereinten Nationen liegt eine konkrete Langfristorientierung vor, und zwar sowohl für den globalen Süden als auch für den globalen Norden (UN 2015b). Die SDG werden die politische Debatte der nächsten eineinhalb Jahrzehnte prägen. In diesem Kontext wird die Möglichkeit bestehen, Ziele wie Klimaschutz, Inklusion, Bekämpfung von Armut und Hunger mit den verschiedenen Aspekten von Postwachstum und globaler Ge- rechtigkeit zusammen zu denken. Dabei zeigt gerade der Entwicklungsprozess auf UN-Ebene von den im Jahr 2000 verabschiedeten, primär die Entwicklungsländer adressierenden Millennium Development Goals (MDG) hin zu den für die gesamte Staatengemeinschaft formulierten SDGs, dass den Aspekten globaler Verantwortung und Gerechtigkeit bezogen auf die Verursachung und Lösung von Problemen eine wachsende Bedeutung zukommt.

Zweitens ist der ganzheitliche, integrative Blick auf den globalen Norden und Süden, auf Entwicklungs-, Wirtschafts-, Gesellschafts- und Gerechtigkeitsfragen und deren Wechselbeziehungen - ein Kerngedanke des Nachhaltigkeitsleitbilds - zentral für Perspektiven, die sich mit Gesellschaften jenseits des Wachstums befassen. Auch wenn im Rahmen der engeren Postwachstumsdebatte um Degrowth immer wieder Verbindungen zu Ansätzen des globalen Südens wie Buen Vivir (Walsh 2010) oder Radical Ecological Democracy (Kothari 2014) gesucht werden, so finden deren Zielsetzungen erst durch die SDGs substanzielle Unterstützung und sind dadurch auf eine breitere institutionelle Basis gestellt.

Drittens ist mit dem Konzept einer Nachhaltigen Entwicklung nicht nur ein politischer Handlungsrahmen aufgespannt, sondern es beschreibt auch eine globale Ethik. Im BrundtlandBericht kommt dies deutlich zum Ausdruck und in der Erklärung von Rio 1992 wird Nachhaltigkeit kaum verkappt als Menschenrecht formuliert. Im Zentrum steht der gerechte Zugang zu natürlichen und gesellschaftlichen Ressourcen für alle Menschen, heute und in Zukunft. Nachhaltige Entwicklung erweitert damit die Reichweite und den Rahmen der Menschenrechte für die Kontexte des 21. Jahrhunderts. Gerade diese globale ethische Dimension Nachhaltiger Entwicklung ist für die breitere Postwachstumsdiskussion wichtig, enthält doch gerade die engere Degrowth-Perspektive immer auch einen gewissen Rückzug auf das Lokale, eine „Ent-Globalisierung" der Verhältnisse und weist damit eine ungute Nähe zu manch anti-modernistischen, anti-globalistischen Strömungen unserer Zeit auf. 


\subsection{Zählt nur die Begrifflichkeit? Die Kontroverse zwischen Stirling und Kallis}

In einer im Februar 2016 geführten öffentlichen Auseinandersetzung zwischen Andrew Stirling und Giorgos Kallis ${ }^{1}$ finden sich paradigmatisch die Unterschiede und Gemeinsamkeiten beider Ansätze. In seinen Beiträgen unter dem Oberbegriff „Outgrowing the Twin Simplifications of Growth and Degrowth" kritisiert Stirling die sprachliche und konzeptionelle Begrenztheit des Degrowth-Begriffes durch den starken Bezug auf wirtschaftliches Wachstum (Stirling 2016). Er verweist darauf, dass die Entwicklung eines diametralen Gegenkonzeptes zu wirtschaftlichem Wachstum, wie es die Degrowthdebatte inhärent macht, letztlich die Gefahr in sich birgt, so Stirling, diese wiederum zu verstärken:

„These impressions risk subconsciously entrenching precisely the kinds of 'no alternatives' fatalism that the hegemony itself imposes."

Dann verweist er darauf, dass eine Transformation in Richtung Nachhaltigkeit letztlich nicht ohne Wachstum in ,bestimmten Praktiken, Technologien und Sektoren" (eigene Übersetzung) möglich sein wird. Stirling spezifiziert diese Wachstumsbereiche im Folgenden als:

„peaceful dispute resolution; open source seeds; ecological farming; collective land tenure; co-operative enterprises; renewable energy; community utilities; grassroots innovation."

Er verweist darauf, dass die Betonung materieller Parameter in der Debatte den Blick dafür verschließt, dass es für eine gesellschaftliche Transformation eines entsprechenden politischen Klimas bedarf, um die Vielfalt sozialer Werte zur Entfaltung zu bringen. Er fordert daher, den Blick dafür zu öffnen, dass
„,more diverse appreciations for the many kinds of possibly ways and values that societies might grow... and the radically different ways of growing. It is in enabling associated plural spac- es for political creativity, contestation and ex- perimentation, that hopes for emancipation lie."

Der Begriff „Degrowth“ sei ein „simplifiziertes, reduktives Schlagwort" (eigene Übersetzung), das die Gefahr berge, die genannten Potenziale zu verdecken. Gerade die Sustainable Development Goals würden mit Einwänden aus Sicht von Stirling einen guten Startpunkt dafür darstellen, denn in ihnen sind nicht-monetäre Werte festgelegt, die weiterentwickelt werden sollen. Letztlich plädiert Stirling dafür, ein plurales Verständnis von Wachstum zu entwickeln, das z. B. auch nicht ausschließt, dass exkludierte Gemeinschaften ebenso wie marginalisierte grüne Infrastrukturen wachsen können.

„The choices lie not just in 'growth or degrowth', but in vibrant democratic struggles for 'many-growths" ".

Um dieses Spannungsfeld aufzuheben, schlägt er schließlich den Begriff „Outgrowth“" vor. Er adressiert damit einen Punkt, der in deliberativen Gesellschaften zentral ist, nämlich die Möglichkeit, in partizipativen und öffentlichen Prozessen ein Spektrum von Wahlmöglichkeiten für Entwicklungspfade zu identifizieren, zu diskutieren und am Ende eine Entscheidung zu treffen. Eine solche kritische Sicht auf die Debatte um Wachstum, grünes Wachstum und Post-Wachstum wird auch von anderen AutorInnen vertreten, beispielsweise aus ökonomischer Perspektive Jakob/Edenhofer (2014), die eine erweiterte Definition von sozialer Wohlfahrt als eine mittlere Position zwischen Green Growth und Degrowth vorschlagen.

Kallis entgegnet unter der Überschrift „Why We Need Degrowth“, dass es seiner Ansicht nach keine Alternative zur Degrowth-Kritik am grenzenlosen wirtschaftlichem Wachstum gibt, den er als ,größte tödliche Bedrohung für die gedeihende Entwicklung der Menschheit auf diesem Planeten“ (eigene Übersetzung) identifiziert (Kallis 2016). Dabei betont er, dass „Degrowth“ qualitative Formen des Wachstums nicht ausschließen würde, sondern sich selbst vielmehr als „Garten der Alternativen“ oder "Ideennetzwerk“ (eigene Übersetzung) versteht. Er verweist vielmehr darauf, dass man für diese sozialen Werte andere Begrifflichkeiten (und Konzepte) als "Growth“ verwenden sollte:

„Equality has to be achieved (or approximated), not grow; Same for justice, sustainability or liberty. Fulfilment, 'human flourishing', or experiences to 'grow'? [...] In all these domains we are talking of qualitative changes, patterns of emergence, change and evolution, not growth." 
Auch bekräftigt er die Unvereinbarkeit von Nachhaltigkeitszielen mit Degrowth gerade anhand der SDGs und fordert vielmehr eine radikale Strategie des Wandels:

„The 'Sustainable Development Goals' Andy applauds shy away from dealing with the radical social reorganization that will be necessary in order to flourish in a world without growth and with radically less material and energy use."

Interessant an diesem Austausch ist, dass beide nicht müde werden, den gegenseitigen Respekt und ihre gemeinsame Position für die ,gute“ künftige Entwicklung zu betonen, und so erscheint es an manchen Stellen, als ob sich der ganze Disput zwischen den beiden nur an der Begrifflichkeit „Growth/Degrowth/Outgrowth“ entzünden würde und ansonsten alles in „bester Ordnung“ wäre. Allerdings zeigt sich in diesem Austausch auch sehr deutlich, dass es letztlich auch um die akademische und politische „Deutungsmacht" für den „richtigen“ Begriff der Transformation in eine bessere Zukunft zu gehen scheint. Während Stirling für eine Integration des DegrowthGedankens in eine gesellschaftliche Transformationsstrategie plädiert, sieht Kallis den einzig richtigen Weg in der Verfolgung einer radikalen „Degrowth-Strategie“, in die er wiederum Stirlings intellektuellen Beitrag integrieren möchte.

\subsection{In der Praxis nicht zu trennen? Die Rolle des Postwachstumsdiskurses innerhalb urbaner Nachhaltigkeitspraxis}

Am Beispiel zweier Stadtforschungsprojekte wird im Folgenden die enge Verknüpfung von Nachhaltigkeits- und Postwachstumsprojekten aufgezeigt. Dabei wird die These verfolgt, dass sowohl Nachhaltige Entwicklung als auch Postwachstum keine gegensätzlichen Forschungsbereiche sind, sondern diese in Wechselwirkung zueinanderstehen und integrativ betrachtet werden können. Im vorliegenden Fall dienen dabei sog. Reallabore als Forschungsformate (Schneidewind/Scheck 2013; Schneidewind 2014; MWK 2013; Wagner/Grunwald 2015), in denen neben Nachhaltigkeitsaktivitäten auch Postwachstumsstrategien sowohl praktisch erprobt als auch deren Wirkweisen aus wissenschaftlich-analytischer Sicht erforscht werden. Die hierfür notwendigen
Ansätze und Methoden stammen beispielsweise aus der transdisziplinären Forschung (Bergmann et al. 2010). Beide Stadtforschungsprojekte orientieren sich dabei an dem integrativen Nachhaltigkeitskonzept der Helmholtz-Gemeinschaft (Kopfmüller et al. 2001). Das ethische Leitbild einer Nachhaltigen Entwicklung bildet demnach den normativen Rahmen für Postwachstumsaktivitäten in städtischen Reallaboren.

Derzeit werden zwei solcher Reallabore von Instituten des KIT unter Federführung des ITAS und in Kooperation mit lokalen nichtakademischen Akteuren in der Karlsruher Oststadt betrieben: Das „Quartier Zukunft - Labor Stadt“ sowie das „Reallabor 131: KIT findet Stadt“. Die in den Reallaboren laufenden Forschungsthemen und -aktivitäten sollen letztendlich die Nachhaltige Entwicklung der Karlsruher Oststadt unterstützen und darüber hinaus allgemeines System-, Ziel-, und Handlungswissen für weitere Kontexte schaffen.

Im Folgenden sollen einige Forschungsthemen und -aktivitäten innerhalb der beiden Reallabore diskutiert werden, die einen wachstumskritischen Bezug aufweisen, postwachstumsnahe Themen behandeln oder eine direkte Verbindung zwischen Nachhaltiger Entwicklung und Postwachstumsdiskurs erkennen lassen.

Partizipation und bürgerliches Engagement spielen eine zentrale Rolle innerhalb von Reallaboren und des Nachhaltigkeitsdiskurses. Es existiert aber eine ökonomische Ungleichheit zwischen unbezahlten ehrenamtlich Tätigen, die im Projekt aktiv beteiligt sind und bezahlten Mitarbeitern, wie beispielsweise den involvierten WissenschaftlerInnen. Um ihre ökonomischen Bedürfnisse decken zu können, sind die Freiwilligen deshalb auf finanzielle Ressourcen aus anderen Tätigkeiten angewiesen. Jedoch sind diese Tätigkeiten in vielen Fällen an Wachstumsprozesse gekoppelt und nicht nachhaltig. Sich mit diesem Dilemma auseinander zu setzen und im Sinne einer Nachhaltigen Entwicklung im Quartier Gegenmodelle zu entwickeln sowie zu erproben, ist ein grundsätzliches Forschungsanliegen innerhalb der beiden Reallaborprojekte. Als Beispiele können die vielfältigen Aktivitäten des bereits etablierten ReperaturCafés genannt werden, 
dessen Rolle im Beitrag von Waitz/Meyer-Soylu in diesem Heft ausführlich erläutert wird.

Zudem tragen beide Reallaborprojekte zur Vernetzung bereits bestehender Initiativen bei, wie Regionalwährung, Gemeinwohlökonomie oder Food Sharing, die an Postwachstumsthemen angeschlossen werden können und auf ihre Wirkung hinsichtlich einer nachhaltigen Quartiersentwicklung untersucht werden. Weitere postwachstumsnahe Fragestellungen ergeben sich außerdem bei der möglichst nachhaltigen Ausgestaltung des Quartierbüros mit Gebrauchsgütern und Dienstleistungen. Dabei wurde besonderer Wert auf Ressourcenschonung, Wiederverwertung sowie auf regionale Wirtschaftskreisläufe gelegt.

Schließlich wird innerhalb der transdisziplinären Realexperimente ein breites Spektrum an Postwachstumsthemen im Quartier abgedeckt. Diese Forschungsaktivitäten sollen in erster Linie der Nachhaltigen Entwicklung des Untersuchungsraums zu Gute kommen, weisen aber an vielerlei Stellen Bezüge zum Postwachstumsdiskurs auf. Hierzu zählt unter anderem die Einrichtung eines Beratungsbüros für Alltagsmobilität, welches bedarfsgerecht die persönliche Mobilität im Quartier verbessern soll und letztendlich die Reduktion des individuellen Kfz-Bestands im Blick hat.

Im Zuge der Reallabordebatte lassen sich auch die bereits genannten Kritikpunkte am Nachhaltigkeitsleitbild seitens der Degrowth-Community aufgreifen (Kap. 3). Es ist richtig, dass die weitreichende Umsetzung einer Nachhaltigen Entwicklung bisher ausblieb. Ein Grund hierfür ist auch, dass bis dato noch zu wenig Wissen über die hierfür notwendigen Transformationsprozesse vorliegt. Durch ihren experimentellen Charakter sollen Reallabore hierzu einen Beitrag leisten. Rebound-Effekte, welche beispielsweise in der Praxis der Sharing Economy auftreten, zeigen jedoch, dass der geschilderte Forschungsbedarf auch auf den Postwachstumsdiskurs zutrifft. Das in Reallaboren erzielte Transformationswissen kommt demnach der Postwachstums- und der Nachhaltigkeitsdebatte zugute.

Es wurde zudem darauf hingewiesen, dass der zu Recht kritisierte Entwicklungsbegriff inhaltlich ausgestaltet und geschärft werden muss, hin zu einer gerechteren Entwicklungspolitik. In diesem Zusammenhang sind jedoch grundsätzliche Diskussionen zum Thema Suffizienz nötig, um nicht nur die gerechte Verteilung, sondern auch den tatsächlichen Bedarf in den Blick zu nehmen. Die Frage nach „Was ist genug“, ist richtungsweisend sowohl für eine Nachhaltige Entwicklung als auch für den Postwachstumsdiskurs und spielt deshalb eine wesentliche Rolle innerhalb der vorgestellten Forschungsprojekte.

Mit Blick auf Reallabore als Forschungsformat zeigt sich, dass Postwachstums- und Nachhaltigkeitsaktivitäten sowie die entsprechenden Forschungsfragen nicht klar voneinander zu trennen sind. Eine mögliche Verknüpfungsoption würde hier darin bestehen, Postwachstumsstrategien als Beitrag zu einer Nachhaltigen Entwicklung zu verstehen, der an den Zielen und Regeln einer Nachhaltigen Entwicklung zu orientieren wäre.

\section{Quo Vadis?}

Anhand der hier vorgenommenen Betrachtungen sollte zum einen verdeutlicht werden, dass die Kritik an der nachhaltigen Entwicklung von Seiten der Postwachstums-Community an vielen Stellen zu einseitig und wenig ausdifferenziert ist. Letztlich unterscheiden ihre Protagonisten zu wenig zwischen wissenschaftlichen oder gesellschaftlichen Leitbildern (wie der nachhaltigen Entwicklung) und der mangelnden bzw. defizitären politischen Umsetzung derselben - ein entscheidender Punkt, wenn es um die Frage geht, weswegen viele Menschheitsprobleme bislang nur unzureichend gelöst worden sind. Dies liegt weniger an Defiziten vorhandener Nachhaltigkeitskonzepte, von denen zumindest einige Grenzen des Wachstums als Teil ihres Ansatzes definiert haben (z. B. Grunwald/Kopfmüller 2012), als vielmehr an der unzureichenden politischgesellschaftlichen Umsetzung. Man könnte umgekehrt - zugegeben ein wenig polemisch - auch fragen, wo denn nach über 15 Jahren Debatten um und über Postwachstum die gesellschaftlich breit getragenen und erfolgreich durchgeführten Beispiele zu finden sind, die über mehr oder weniger kleinräumige Nischenaktivitäten hinausgehen.

Zum anderen wurde gezeigt, dass die beiden Debatten starke inhaltliche Überschneidungen und geteilte Ziele aufweisen, auch wenn sie 
vermutlich auch in Zukunft getrennt voneinander geführt werden. Allerdings wurde hier wie auch in anderen Beiträgen des Schwerpunkts gezeigt, dass diese Unterscheidung sowohl auf konzeptioneller, praktischer als auch auf politischer Ebene (Ober in diesem Heft) nicht immer nachzuvollziehen ist. Die im Rahmen des anlaufenden Prozesses zur Umsetzung der SDGs diskutierten Ansätze etwa einer auf globaler Gerechtigkeit beruhenden Global Governance bergen zumindest das Potenzial, die Gemeinsamkeiten beider Leitbilder herauszuarbeiten und nutzbar zu machen. Die Auseinandersetzung von Stirling und Kallis zeigt die gemeinsamen Ziele beider Bewegungen auf, was auch für die geschilderten Erfahrungen aus den Reallabor-Projekten in Karlsruhe gilt, die sich als offen für Postwachstumsstrategien verstehen, sich aber primär innerhalb der urbanen Nachhaltigkeitspraxis verorten. Hierbei ist auch anzumerken, dass Reallabore mitunter explizit als Teil einer Postwachstumsstrategie gesehen werden (Eversberg/Schmelzer 2016).

Dabei gibt es letztlich durchaus Argumente dafür, die Trennung beider Debatten zu belassen, da sich aus der Abgrenzung durchaus fruchtbare Debatten und Erkenntnisse ziehen lassen. So ist auf der einen Seite die Bewahrung der Radikalität der Degrowth-Debatte durchaus ein Argument dafür, beide Debatten nicht zu stark zu vermischen (Flipo/Schneider 2015). Denn radikale Positionen erlauben es, frische Ideen in womöglich eingefahrene politische wie auch wissenschaftliche Debatten zu bringen und dabei immer wieder den Blick auf mögliche Alternativen zu richten, womit letztlich möglicherweise „mehr“ erreicht werden kann als ohne eine solche aus Radikalität resultierende Aufmerksamkeit dafür. Auch kann durch zwei getrennt „marschierende“ Bewegungen durchaus eine höhere öffentliche oder politische Aufmerksamkeit erreicht werden. Auf der anderen Seite wird durch die Abgrenzung möglicherweise viel Potenzial verschenkt, das im Einsatz für das Erreichen geteilter, weltweiter Ziele der ,großen Transformation" dringend notwendig wäre. Die bereits angeführte Forschung zu Postwachstumsunternehmen zeigt dabei, dass auch vermeintlich „wachstumsfreundliche“ Akteure durchaus anders agieren und Zugänge zum Postwachstumsdiskurs aufgebaut werden können. So hat eine Umfrage des IÖW unter deutschen KMUS ergeben, dass bei einem Drittel Wachstumsziele keine Rolle spielen. Gut die Hälfte setzt auf weniger starkes Wachstum (Gebauer/Sagebiel 2015). Gerade die Perspektive solcher Akteure, die bislang wenig sichtbar sind, kann einen Gewinn für den Postwachstumsdiskurs sein und weitere gesellschaftliche Anschlüsse eröffnen.

Allerdings sollte künftig eine sorgfältigere Reflexion und Diskussion über Gründe der Trennung beider Debatten und mögliche Nutzen der Schärfung von Gemeinsamkeiten erfolgen, denn jenseits terminologischer Differenzen sollte es letztlich auf gesellschaftlicher Ebene darum gehen, Ziele und mögliche Wege in eine von beiden Bewegungen angestrebte Zukunft zu eruieren. So ist durchaus anzunehmen, dass ein Blick in die Geschichte der Nachhaltigkeitsdebatte zu Erkenntnisgewinnen auch für die Degrowth-Bewegung führen kann. Gleichzeitig profitiert die Nachhaltigkeitsdebatte vom kritischen Ansatz der Degrowth-Bewegung. Beispielsweise könnte die im Verlauf der Nachhaltigkeitsdebatte in den Hintergrund getretene Strategie der Suffizienz mit der Degrowth-Debatte wieder stärkere Aufmerksamkeit erlangen, und zwar nicht als individuelle Entscheidung und Verantwortung (z. B. aus Sicht des ,nachhaltigen Konsums“), sondern als gesellschaftliche und politische Frage des „nicht mehr haben wollen Müssens“ (Winterfeldt 2007). Aus all dem kann verschiedentlicher Forschungsbedarf abgeleitet werden: Für die Degrowth-Bewegung könnte in einer historischen Perspektive nachvollzogen werden, welche Faktoren, Einflüsse und Diskursformationen letztlich dazu geführt haben, dass sich diese Strategie des Weniger bislang nicht durchgesetzt hat. Für die Nachhaltigkeitsforschung könnte die kritische Frage gestellt werden, an welchen Stellen sie zu integrativ war, d. h. zu viele Handlungsoptionen zugelassen hat, sodass die Effizienzstrategie eine dominierende Rolle gegenüber Suffizienzüberlegungen einnehmen konnte.

Hierfür ist ein offener Diskurs und Austausch zwischen beiden Konzepten erforderlich, der beiden Communities neue Anregungen liefern kann. Um diesen anzustoßen, könnte eine Möglichkeit darin bestehen, das Verständnis von wachstumskritischen Ansätzen durch weitere 
Postwachstumsansätze und Perspektiven zu erweitern, die in Relation zur Degrowth-Bewegung gesetzt werden und möglicherweise Hinweise für eine Neujustierung und Klärung des Verhältnisses zum Wachstumsbegriff auch für die Nachhaltige Entwicklung geben können. Für Postwachstum im weiteren Sinne, wie eingangs definiert, bietet sich dann die Möglichkeit, sich - im Sinne der SDG - stärker mit globalen Fragen zu befassen und auch spezifische Postwachstumsansätze für die globale Ebene zu entwickeln. Hierbei könnten z. B. bislang unbeantwortete Fragen adressiert werden, etwa wie eine globale Lastenverteilung von Postwachstumsstrategien aussehen könnte, wie Ziele, Ausprägungen und Folgen von solchen Strategien in einem nationalen aber auch globalen Kontext zu sehen wären oder welche gesellschaftlichen Diskurse hierfür zu führen wären. Mit einer solchen Auseinandersetzung könnte die Grundlage dafür geschaffen werden, sich - gemeinsam mit den VertreterInnen des Nachhaltigkeitsleitbilds - als Teil eines globalen politischen und ethischen Diskurses zu verstehen, eine gemeinsame Zukunft zu begreifen und entsprechend wirksam zu werden.

\section{Anmerkung}

1) Andrew Stirling ist Professor für „Science \& Technology Policy“ bei SPRU (Science Policy Research Unit) an der School of Business, Management and Economics in Sussex, UK. Er forscht im Feld der „sustainability transitions“. Giorgos Kallis ist Professor für Social \& Behavioural Sciences an der Autonomous University in Barcelona, Spanien, und forscht im Feld der DegrowthBewegung.

\section{Literatur}

Bergmann, M.; Jahn, T.; Knobloch, T. et al., 2010: Methoden transdisziplinärer Forschung. Ein Überblick mit Anwendungsbeispielen. Frankfurt a. M.

Binswanger, M.; Beltrani, G.; Jochem, A. et al., 2005: Wachstum und Umweltbelastung: Findet eine Entkopplung statt? Bern

D'Alisa, G.; Demaria, F.; Kallis, G., 2015: Preface. In: D'Alisa, G.; Demaria, F.; Kallis, G. (Hg.): Degrowth. A Vocabulary for a New Era. New York, S. xx-xxii
Daly, H.; Farley, J., 2003: Ecological Economics: Principles and Application. Washington

Eversberg, D.; Schmelzer, M., 2016: Über die Selbstproblematisierung zur Kapitalismuskritik. Vier Thesen zur entstehenden Degrowth-Bewegung. In: Forschungsjournal Soziale Bewegungen 1 (2016), S. 9-16 Flipo, F.; Schneider, F., 2015: Foreword. In: D’Alisa, G.; Demaria, F.; Kallis, G. (Hg.): Degrowth. A Vocabulary for a New Era. New York, S. xxiii-xxvi

Group of 77, 2015: Ministerial Declaration; http:// www.g77.org/doc/Declaration2015.htm (download 13.7.16)

Grunwald, A., 2015: Ökomodernismus ist verantwortungsethisch nicht haltbar. In: GAIA - Ecological Perspectives for Science and Society 24/4 (2015), S. 249-253; doi: 10.14512/gaia.24.4.10

Grunwald, A.; Kopfmüller, J., 2012: Nachhaltigkeit. Frankfurt a. M.

Hinton, J.; Maclurcan, D., 2014: How on Earth: Flourishing in a Not-for-Profit World by 2050. Burlington

Huber, J., 1995: Nachhaltige Entwicklung. Strategien für eine ökologische und soziale Erdpolitik. Berlin

Jakob, M.; Edenhofer, O., 2014: Green Growth, Degrowth, and the Commons. Oxford Review of Economic Policy 30/3 (2014), S. 447-468

Kallis, G., 2016: Why We Need Degrowth. In: Blog des STEPS Centre; http://steps-centre.org/2016/blog/ why-we-need-degrowth/ (download 13.7.16)

Kopfmüller, J.; Brandl, V.; Jörissen, J. et al., 2001: Nachhaltige Entwicklung integrativ betrachtet. Konstitutive Elemente, Regeln, Indikatoren. Berlin

Latouche, S., 2004: Degrowth Economics: Why Less Should be Much More. In: Le Monde Diplomatique November 2004, Paris; http://mondediplo. com/2004/11/14latouche (download 20.7.16)

Liesen, A.; Dietsche, C.; Gebauer, J., 2013: Wachstumsneutrale Unternehmen: Pilotstudie zur Unternehmensperspektive im Postwachstumsdiskurs. Berlin

Martinez-Alier, J.; Pascual, U.; Vivien, F. et al., 2010: Sustainable De-growth: Mapping the Context, Criticisms and Future Prospects of an Emergent Paradigm. In: Ecological Economics 69 (2010), S. 1741-1747; DOI: 10.1016/j.ecolecon.2010.04.017

MWK - Ministerium für Wissenschaft, Forschung und Kunst Baden-Württemberg, 2013: Wissenschaft für Nachhaltigkeit. Herausforderung und Chance für das baden-württembergische Wissenschaftssystem. Expertengruppe „Wissenschaft für Nachhaltigkeit“ - Bericht. Unter Mitarbeit von Uwe Schneidewind und Karin Boschert. Stuttgart; https://mwk. baden-wuerttemberg.de/fileadmin/redaktion/m- 
mwk/intern/dateien/pdf/Wissenschaft_f $\% \mathrm{C} 3 \% \mathrm{BCr}$ Nachhaltigkeit/Expertenbericht_RZ_MWK Broschuere_Nachhaltigkeit_Web.pdf (download 22.7.16)

Paech, N., 2005: Nachhaltiges Wirtschaften jenseits von Innovationsorientierung und Wachstum: Eine unternehmensbezogene Transformationstheorie. Marburg Paech, N., 2009: Postwachstumsökonomie - Ein Vademecum. In: Zeitschrift für Sozialökonomie 46/160 161 (2009), S. 28-31

Post Growth Institute, 2016: About Post Growth; http://postgrowth.org/learn/about-post-growth/ (download 12.7.16)

Reichel, A., 2013: Das Ende des Wirtschaftswachstums, wie wir es kennen: Betriebswirtschaftliche Perspektiven auf die Postwachstumsökonomie. In: Ökologisches Wirtschaften 27/1 (2013), S. 15-18

Research \& Degrowth, 2016: Definition; http://www. degrowth.org/definition-2 (download 12.7.16)

Rockström, J.; Steffen, W.; Noone, K. et al., 2009: A Safe Operating Space for Humanity. In: Nature 461 (2009), S. 472-475; doi: 10.1038/461472a

Schneidewind, U., 2014: Urbane Reallabore - ein Blick in die aktuelle Forschungswerkstatt. In: pnd - online 3 (2014), S. 1-7; http://www.planung-neudenken.de/images/stories/pnd/dokumente/3_2014/ schneidewind.pdf (download 25.7.16)

Schneidewind, U.; Scheck, H., 2013: Die Stadt als Reallabor für Systeminnovationen. In: Rückert-John, J. (Hg.): Soziale Innovation und Nachhaltigkeit. Perspektiven sozialen Wandels. Wiesbaden, S. 229-248

Spaeth, J., 2009: Doing Business in a Postgrowth Society. In: Harvard Business Review 87/9 (2009), S. 18-19

Stirling, A., 2016: Outgrowing the Twin Simplifications of Growth and Degrowth. In: Blog des STEPS Centre; http://steps-centre.org/2016/blog/outgrowing-the-twin-simplifications-of-growth-and-degrowth-part-1 bis part-3/ (download 22.6.16)

Stockmann, R.; Menzel, U.; Nuscheler, F., 2015: Entwicklungspolitik: Theorien - Probleme - Strategien. Berlin

Truman, H., 1949: Inaugural Address. January 20, 1949; https://www.trumanlibrary.org/whistlestop/50yr archive/inagural20jan1949.htm (download 12.7.16)

UN - United Nations, 2015a: Millenniums-Entwicklungsziele. Bericht 2015. New York

UN - United Nations, 2015b: Sustainable Development Goals; https://sustainabledevelopment.un.org/ sdgs (download 13.7.16)
UN-ESCAP - United Nations Economic and Social Commission for Asia and the Pacific, 2009: What is Good Governance? Bangkok

Wagner, F.; Grunwald, A., 2015: Reallabore als Forschungs- und Transformationsinstrument. Die Quadratur des hermeneutischen Zirkels. In: GAIA - Ecological Perspectives for Science and Society 24/1 (2015), S. 26-31; DOI: 10.14512/gaia.24.1.7

Walsh, C., 2010: Development as Buen Vivir: Institutional Arrangements and (De)Colonial Entanglements. In: Development 53/1 (2010), S.15-21

WCED - World Commission on Environment and Development, 1987: Our Common Future. Oxford Willke, H., 2006: Global Governance. Bielefeld

Winterfeldt, U. von, 2007: Keine Nachhaltigkeit ohne Suffizienz: Fünf Thesen und Folgerungen. In: Vorgänge 46/3 (2007), S. 46-54

WWF - World Wildlife Fund, 2014: Living Planet Report 2014. Gland

Zürn, M., 1998: Regieren jenseits des Nationalstaates. Frankfurt a. M.

\section{Kontakt}

Jürgen Kopfmüller Institut für Technikfolgenabschätzung und Systemanalyse (ITAS)

Karlsruher Institut für Technologie (KIT)

Karlstraße 11, 76133 Karlsruhe

Tel.: +49 721 608-24570

E-Mail: juergen.kopfmueller@kit edu 